\title{
Morphological Hierarchical Segmentation and Color Spaces
}

\author{
Cyril Meurie, ${ }^{1}$ Olivier Lezoray, ${ }^{2}$ Louahdi Khoudour, ${ }^{3}$ Abderrahim Elmoataz ${ }^{2}$ \\ ${ }^{1}$ UTBM-SeT, Equipe ICAP, 13 rue Ernest-Thierry Mieg, 90010 Belfort Cedex, France \\ 2 GREYC UMR-CNRS 6072, Equipe Image, 6 Bd Maréchal Juin, 14050 Caen, France \\ ${ }^{3}$ INRETS-LEOST, 20 rue Elisée Reclus, BP 317, 59666 Villeneuve d'Ascq Cedex, France
}

Received 13 June 2008; accepted 2 April 2010

\begin{abstract}
In this article, the color space influence on different methods of morphological hierarchical segmentation is studied. For this purpose, several experimental studies are carried out on hundred images of the "Berkeley Segmentation Dataset and Benchmark" (Berkeley). On one hand, four usual and representative color spaces (RGB, Y $\left.C_{\mathrm{b}} C_{\mathrm{v}} L^{*} \mathrm{a}^{*} b^{*}, I H S L\right)$ are considered. On the other hand, three different morphological hierarchical segmentation schemes are considered. These later are the quasi flat zones, the nonparametric hierarchical watershed (waterfall algorithm) and the nested homogeneous connections. As one could expect it, the different morphological hierarchical segmentation schemes do not have the same behavior in different color spaces. We conclude by proposing a ranking of color spaces according to the considered morphological hierarchical segmentation schemes. ( 2010 Wiley Periodicals, Inc. Int J Imaging Syst Technol, 20, 167-178, 2010; Published online in Wiley InterScience (www.interscience.wiley.com). DOI 10.1002/ima.20233
\end{abstract}

Key words: mathematical morphology; hierarchical segmentation; color image segmentation; color spaces; nested homogenous connections

\section{INTRODUCTION}

Image segmentation consists in partitioning an image in more or less regular or coherent zones according to a given criterion. Thus, one usually seeks an image partition in zones whose values follow a given model of organization. Automatic segmentation of images is a central problem in image processing since a partition of the image in regions makes the extraction of the primary visual components from an image possible; these later being used to identify and recognize objects of interest. However, there is a gap between the image itself and its description as a sole partition of the image into several regions. A way of circumventing this gap is to concentrate on region segmentation on one hand and on perceptual groupings extracted by a hierarchical vision of images on the other hand. The union of regions is a group of elements which is also a region at a

Correspondence to: Cyril Meurie; e-mail: cyril.meurie@utbm.fr given scale, with local inner properties and global ones according to its neighborhood. Mostly, low-level image segmentation algorithms cannot directly cope with this semantic gap as it is very difficult to directly construct the best image partition (if there is one). Thus, it is necessary to deal with hierarchical methods which produce a multiscale image segmentation. In this article, we take a morphological point of view of hierarchical segmentation (Serra, 2006; Meyer, 2001). However, when considering morphological segmentation, one needs to work on complete lattices (Ronse, 1990) and the problems aspects of the classical gray-scale morphological algorithms to color ones arises (Plataniotis and Venetsanopoulos, 1998). This extension is not simple (Angulo, 2005) and still unsolved (Serra, 2005; Lezoray et al., 2005a). A lot of articles in the literature consider the influence of color spaces for simple morphological operations such as erosion or dilatation (Peters, 1997; Chassunot and Lambert, 1998; Talbot, 1998; Comer and Delp, 1999; Ortiz et al., 2001; Tsalides, 2002; Lezoray et al., 2005a). Few works consider the influence of color spaces on sets of nested segmentations generated by morphological hierarchical segmentation approaches (Angulo and Marcotegui, 2005). Therefore, we propose a more in-depth study of the color space influence on several different morphological hierarchical segmentation schemes all based on the connective criterion principle of Serra (Serra, 2006). The article is organized as follows. In Section II, we recall the basic relations allowing to shift from the $R G B$ or the $X Y Z$ color spaces to a given color space among the ones we selected $\left(Y C_{\mathrm{b}} C_{\mathrm{r}}, L^{*} a^{*} b^{*}, I H S L\right)$. In Section III, the principles of morphological hierarchical segmentation are detailed and three classical connective criteria are reviewed: the quasi flat zones (Salembier and Serra, 1992; Crespo et al., 1997; Meyer, 1998), the nonparametric hierarchical watershed: waterfall algorithm (Beucher, 1994; Angulo and Serra, 2003), and the nested homogeneous connections (Meurie 2005; Lezoray et al., 2006). To finish, several experimental studies are carried out on hundred images of the "Berkeley Segmentation Dataset and Benchmark: BSDB" (Berkeley) and the color space influence on the three above mentioned methods of morphological hierarchical segmentation are analyzed on the whole database. 


\section{COLOR SPACES}

The main aim of this work is to show the color space influence on different methods of morphological hierarchical segmentation, we recall some basic concepts on color spaces used in this article. To be rather exhaustive without having to test all existing color spaces (Sharma and Trussel, 1997), we used four representative color spaces belonging to the color spaces families described by Vandenbroucke et al.(2000). The definitions of a luminance-chrominance space $\left(Y C_{\mathrm{b}} C_{\mathrm{r}}\right)$, a perceptually uniform color space $\left(L^{*} a^{*} b^{*}\right)$ and a perceptual coordinate color space (IHSL) are recalled.

\section{A. YCbCr COLOR SPACE}

The $Y C_{\mathrm{b}} C_{\mathrm{r}}$ color space (Plataniotis and Venetsanopoulos, 2000; Lukac and Plataniotis, 2006) is the international standard dedicated to the digital coding of numerical television images. It has the particularity to not impose any rule about the reference white comparing to other color spaces dedicated to television. The transformation of $R G B$ into $Y C_{\mathrm{b}} C_{\mathrm{r}}$ color space is provided by the following relation:

$$
\left[\begin{array}{c}
Y \\
C_{\mathrm{b}} \\
C_{\mathrm{r}}
\end{array}\right]=\left[\begin{array}{ccc}
0.2989 & 0.5866 & 0.1145 \\
-0.1688 & -0.3312 & 0.5 \\
0.5 & -0.4184 & -0.0816
\end{array}\right] \times\left[\begin{array}{c}
R \\
G \\
B
\end{array}\right]
$$

B. L*a*b* Color Space. The $L^{*} a^{*} b^{*}$ color space is an approximation of the Adams-Nickerson's color space in which the perceptual amplitude of the color is defined in terms of opposite colors scales covering the total spectrum seen by the human eye. The transformation to the $L^{*} a^{*} b^{*}$ system is obtained by the following nonlinear relations (Wyszecki and Stiles, 1982):

$$
\begin{aligned}
& L^{*}= \begin{cases}116 \times\left(\frac{Y}{Y_{0}}\right)^{\frac{1}{3}}-16 & \text { if } \frac{Y}{Y_{0}}>0.008856 \\
903.3 \times\left(\frac{Y}{Y_{0}}\right) & \text { if } \frac{Y}{Y_{0}} \leq 0.008856\end{cases} \\
& a^{*}=500\left(f\left(\frac{X}{X_{0}}\right)-f\left(\frac{Y}{Y_{0}}\right)\right) \\
& b^{*}=300\left(f\left(\frac{Y}{Y_{0}}\right)-f\left(\frac{Z}{Z_{0}}\right)\right) \\
& f(x)= \begin{cases}x^{\frac{1}{3}}-16 & \text { if } x>0.008856 \\
7.787 x+\left(\frac{16}{116}\right) & \text { if } x \leq 0.008856\end{cases}
\end{aligned}
$$

$L$ denotes the luminance and the black-white opposition by a value between 0 (black) and 100 (white). $a^{*}$ measures the green-red opposition by a value between -100 and $+100\left(a^{*}\right.$ is positive if the color contains red, negative if the color contains green and null if none of both). $b^{*}$ measures the blue-yellow opposition by a value between -100 and +100 ( $b^{*}$ is positive if the color contains yellow, negative if the color contains blue and null if none of both). $X_{0}, Y_{0}, Z_{0}$ denotes the $X Y Z$ coordinates of the illuminate (illuminate $E$ for our study).

C. IHSL Color Space. The IHSL color space has been proposed by (Hanbury and Serra, 2001a; Hanbury and Serra, 2001b; Hanbury, 2003 ) is an improvement of the HSI color space to overcome its limitations [see in (Hanbury and Serra, 2001a; Hanbury and Serra, 2001b; Hanbury, 2003) for further details]. This color space is now extensively used in mathematical morphology (see in Angulo, 2005) for an in-depth complete review). The transformation of $R G B$ into the IHSL color space is provided by the following relations:

$$
\begin{aligned}
& L=0.2126 \times R+0.7152 \times G+0.0722 \times B \\
& S=\max (R, G, B)-\min (R, G, B) \\
& H=\left\{\begin{array}{c}
360^{\circ}-H_{1} \text { if } B>G \\
H_{1} \text { if } B<G \\
H_{1}=\arccos \left[\frac{R-\frac{(G+B)}{2}}{\left(R^{2}+G^{2}+B^{2}-R G-R B-B G\right)^{\frac{1}{2}}}\right]
\end{array}\right.
\end{aligned}
$$

III. Hierarchical Segmentation of Color Images. In this section, the principles of morphological hierarchical segmentation are recalled (Meyer, 2001a,b). We then focus on the definition of connective criterion and recall two connections: threshold connections [quasi flat zones (Salembier and Serra, 1992; Crespo et al., 1997; Meyer, 1998)] and pathwise connections (nonparametric hierarchical watershed also called waterfall (Beucher, 1994; Angulo and Serra, 2003). Finally, we present a criterion introduced in (Lezoray et al., 2005b; Meurie, 2005; Lezoray et al., 2006), and which extracts the homogeneous zones of an image.

A. Partition of Image. The traditional problem of the automatic segmentation of images is generally considered like a division of the image in disjoined areas, the result being a partition of the image domain. An image $I$ is a set of pixels $I=\left\{p_{1}, p_{2}, \ldots, p_{n}\right\}$ and a region $R$ is a subset of the image pixels composed of $|R|$ pixels. Regions are therefore sets of pixels which usually fulfill a given homogeneity criterion.

Definition 1: Partition (Zucker, 1976). A partition $P$ is a set of regions $P=\left\{R_{1}, R_{2}, \ldots, R_{k}\right\}$ so that:

1. the union of the partition regions provides the initial set:

$$
\begin{aligned}
& I=\bigcup_{i=1}^{k} R_{i}, \\
& \text { 2. regions are disjoined: } \forall i, j, i \neq j, R_{i} \cap R_{j}=\varnothing
\end{aligned}
$$

Several algorithms can be used to segment an image and thus to create a partition (Serra, 2005). But these algorithms can also define, while exploiting their parameters, a stacking of partitions of increasing levels called a hierarchy of partitions. In mathematical morphology, it is important to define an ordering relation between two partitions to have a complete lattice of partitions: a partition $P$ is included in a partition $Q$ if every regions $R_{j}^{p}$ is completely included in a region $R_{i}^{\mathrm{q}}$. With this property, we can define a hierarchy of nested partitions for an image. Let $H$ be a set of partitions associated to an image, $H$ is a hierarchy of nested partitions if it is possible to establish an inclusion order between any pair of $H$ elements. It means that two regions from two different partitions of a hierarchy are either disjoined or included one in to the other.

Definition 2: Hierarchy of Nested Partitions (Horowitz and Pavlidis, 1976). A hierarchy of nested partitions is a set of partitions $H=\left\{P_{1}, P_{2}, \ldots, P_{l}\right\}$ so that the regions of the partition $P_{i}=\left\{R_{1}^{i}, R_{2}^{i}, \ldots, R_{k}^{i}\right\}$ are all included in the regions of the partition $P_{j}=\left\{R_{1}^{j}, R_{2}^{j}, \ldots, R_{k}^{j}\right\}$ with $j>i, k>k^{\prime}$ and $R_{m}^{i} \subseteq R_{p}^{j}$ or $R_{m}^{i} \cap R_{p}^{j}=\varnothing$.

$P_{i}$ is called the $i$ th level of the hierarchy, $P_{0}$ if the lowest one and is the finest partition, $P_{l}$ is the highest level of the hierarchy and is the coarsest partition. The regions of the lowest level being always included in higher level regions, the regions of the $(i+1)$ th 
partition can be obtained by merging ones of the $i$ th partition. Therefore, a hierarchy of partitions is naturally represented by a stack of region adjacency graphs (RAG) also called an irregular pyramid (Jolion and Montanvert, 1992). Links between regions that merge from one level to the next one are contained in a so-called contraction kernel (Kropatsch, 1995).

In mathematical morphology, to have an ordering relation between successive levels of the hierarchy implies that the later forms a complete lattice. The main morphological criteria that define hierarchies of partitions are based on connections (connective criteria), (Serra, 2000; Serra, 2006). This enables to divide an image into zones according to a given criterion. For instance, an image $I$ is divided into flat and connected zones when a partition $P_{i}$ is created, so that for every $x$, the region $R \in P_{i}$ with $x \in R$ is the highest connected component which includes $x$ and where the image $I$ is constant and always equal to $x$. Whatever the connective criterion, there is always a way of partitioning an image into regions that fulfill that criterion.

Definition 3: Connective criterion (Serra, 2006). A criterion $\sigma: F \times P(E) \rightarrow[0,1]$ is connective when, for each $f \in F$, the sets $A$ such that $\sigma[f, A]=1$ form a connection, i.e.,

1. when $\sigma$ is satisfied on the class $S$ of the singletons and by the empty set, $\forall f \in F,\{x\} \in S \Rightarrow \sigma[f\{x\}]=1$

2. when for any function $f \varepsilon F$ and for all families $\left\{A_{i}\right\}$ of $P(E)$ for which the criterion $\sigma$ is satisfied, we have $\cap A_{i} \neq 0$ and $\wedge^{\wedge} \sigma\left[f, A_{i}\right]=1 \Rightarrow \sigma\left[f, \cup A_{i}\right]=1$

In other words, when a connective criterion $S$ is satisfied by a function $f$ on a family $\left\{A_{i}\right\}$ of regions of the space, and if all these regions have one common point, then it is also satisfied on the union $\cup A_{i}$. A zone $A$ is said to be homogeneous according to $f$ and $\sigma$ when $\sigma[f, A]=1$.

Definition 4: Connective Criterion for Segmentation (Serra, 2006). Let us consider two arbitrarily sets $E$ and $T$, and a family $F$ of functions $f: E \rightarrow T$ and let $\sigma$ be a criterion on class $F$. Then the three following statements are equivalent:

1. Criterion $\sigma$ is connective,

2. For each function $f \in F$, those sets on which criterion $\sigma$ is satisfied constitute a connection $C, C=\{A / A \in P(E)$ and $\sigma[f, A]=1\}$.

3. Criterion $\sigma$ segments all functions of the family $F$.

In the following subsection, we recall the two main morphological connective criteria for segmentation: the flat zones and the nonparametric hierarchical watershed (waterfall algorithm). A third connective criterion called nested homogeneous connections is presented and whose behavior is in between the two above mentioned.

B. The Flat Zones and Quasi Flat Zones. The flat zones of an image $I$ are the maximal connected components having a constant value and were introduced by Salembier and Serra [(Salembier and Serra, 1992; Crespo et al., 1997). The flat zone concept relies therefore on a threshold connective criterion. Using directly the partition of an image in flat zones is not very interesting because a very over segmented partition is obtained. The extraction of the flat zones of an image is generally accompanied with the use of a preliminary filtering which increases the flat zones to reduce the over segmentation effect. This is the purpose of levelings, proposed by Meyer (Meyer, 1998), which enlarges flat zones according to a marker image. However, even with the use of levelings to simplify the image, small variations still occur (this is even true with color images) and Meyer (Meyer, 1998) has proposed to extend the principle of flat zones to quasi flat zones.

Definition 5: The Quasi-Flat Zones (Meyer, 1998). Two points $p$ and $q$ belong to the same quasi flat zone of an image $I$ if there is a connected path $\left(p_{1}, p_{2}, \ldots, p_{n}\right)$ between those two points so that $p_{1}=p$ and $p_{n}=q$ and for each $i,\left\|I\left(p_{i}\right)-I\left(p_{i+1}\right)\right\|<\beta$

$\|$.$\| is a L_{2}$-norm and increasing values of $\beta$ create a hierarchy of partitions. Quasi flat zones are strict flat zones for a threshold criterion equal to zero $(\beta=0)$. Increasing values of $\beta$ defines a hierarchy of partitions. The progression in the hierarchy levels is strongly correlated with the number of regions and the loss of information. It is therefore important to be careful on the determination of this threshold criterion for obtaining a single efficient segmentation. Figure 1 presents several segmentations produced by the quasi flat zones principle for different hierarchy levels $(\beta \in\{1,5,10\})$ in two color spaces $\left(R G B, L^{*} a^{*} b^{*}\right)$.

C. Nonparametric Hierarchical Watershed. The watershed is a region growing algorithm which defines a pathwise connection. The watershed lines associate a catchment basin to each minimum of a function. Typically, the function to flood is a gradient function which catches the transitions between the regions. Region seeds of the watershed are therefore the gradient minima. Working with color images, the definition of an appropriate color gradient is the core part of the watershed. At this time, there is no consensus on the way to obtain an appropriate color morphological gradient whatever the color space. This limitation is due to the fact that there is no natural total ordering of multivariate data (Barnett, 1976; Pitas and Tsalides, 1991). To have a watershed whose definition remains the same whatever the color space under consideration, a general formulation of a color geometry is needed. In this article, we consider the classical one which was originally proposed by Di Zenzo (Dizenzo, 1986). Other ad hoc formulations usually used in mathematical morphology (Angulo and Serra, 2003) can also be considered but they are color space dependent. That is why, the Di Zenzo's gradient formulation might be preferred since it is now considered as the classical way to compute the gradient of a color image. We will however also consider in the sequel the classical morphological gradients for comparison purposes (Angulo and Serra, 2003). The watershed is extensively used for the segmentation of images but its major drawback relies on the production of over segmented partitions mainly because of the presence of a high number of significant gradient minima. As for flat zones, one can try to overcome this problem by filtering the image, but this comes to report the problem on an appropriate tuning of a given filtering operation. Another solution to disguise these disadvantages consists in using specific markers instead of gradient minima. Whatever the technique used to overcome over segmentation, it introduces an apriori information and therefore techniques of hierarchical segmentation are of interest for the production of a nonparametric hierarchical watershed also called waterfall. The waterfall algorithm (Beucher, 1994; Angulo and Serra, 2003) enables to construct a nonparametric hierarchy of watersheds (a hierarchy of partitions) which performs region merging between adjacent catchment basins. 


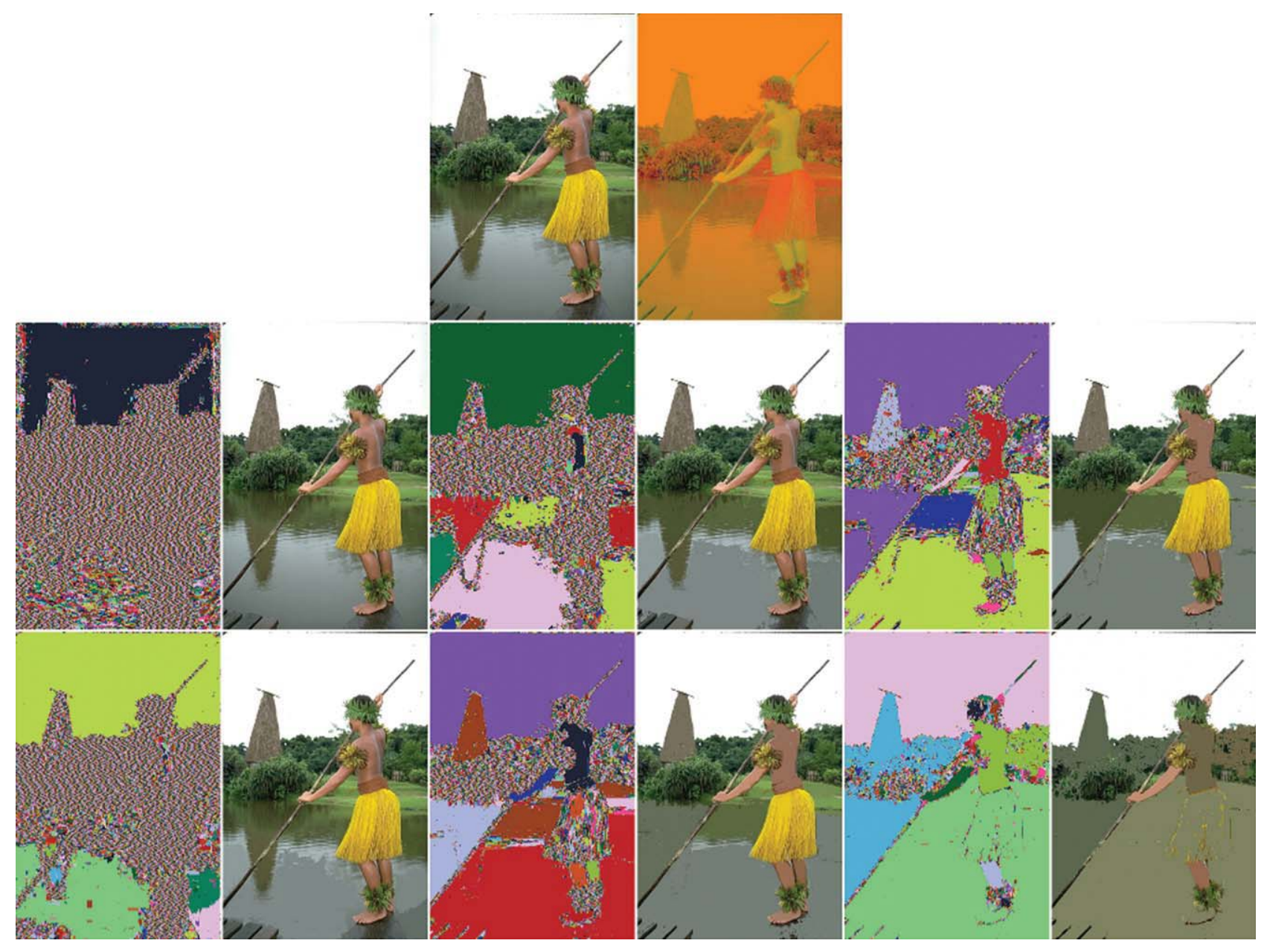

Figure 1. Hierarchies of partitions produced by the quasi flat zones principle at levels (ie. B) $1,5,10$. First line: initial image in $R G B$ and $L^{*} a^{*} b^{*}$, second line: RGB quasi flat zones and their colorized images, third line: $L^{*} a^{*} b^{*}$ quasi flat zones and their colorized images. [Color figure can be viewed in the online issue, which is available at www.interscience.wiley.com.]

It is based on an iterative process which computes the gradient on a specific image called the mosaic image (obtained by associating its color mean value to the catchment basins). By carrying out several times this operation, we obtain a cascade of watersheds i.e., a hierarchy of partitions.

\section{Definition 6: Algorithm of the Nonparametric Hierarchical} Watershed (Beucher, 1994). Let $m_{0}$ be a color image and $g_{0}$ its gradient (positive bounded function $0 \leq g_{\mathrm{o}}(x) \leq M$ ).

1. Let $W\left(g_{i}\right)$ be the watershed of $g_{i}$,

2. Define a new function $h_{i}=\left\{\begin{array}{c}g_{i}(x) \text { if } x \in W\left(g_{i}\right) \\ M \text { otherwise }\end{array}\right.$

3. $g_{i}$ is reconstructed by geodesic erosions from $h_{i}$ i.e., $g_{i}^{*}=\epsilon_{\mathrm{g} i}^{\mathrm{rec}}\left(h_{i}\right)$

4. Compute the watershed of $g_{i} *$ and the corresponding mosaic $m_{i}$. Let $g_{i+1}$ be the gradient of $m_{i}$ and iterate $(i=i+1)$.

Figure 2 presents several segmentations produced by the waterfall algorithm for different levels of the hierarchy $(\beta \in\{1,10,20\})$ in two color spaces $\left(R G B, Y C_{\mathrm{b}} C_{\mathrm{r}}\right)$ with the Di Zenzo gradient.
D. Nested Homogeneous Connections. Flat zones hierarchies are usually too fine and waterfall ones too coarse; thus, we present a connective criterion which is an intermediate one between pathwise connections (watersheds) and threshold connections (quasi flat zones). This new criterion is referred to as homogeneous connections and was introduced by Lezoray et al., (2005b; Meurie, 2005; Lezoray et al., 2006).

Definition 7: Homogeneous Connections (Lezoray et al., 2006). Two points $p$ and $q$ belong to a same homogeneous zone of an image $I$ if

$\|I(p)-I(q)\| \leq k \times \beta(\operatorname{Seed}(p))$ with $\operatorname{Seed}(p)$ the initial seed of the region of $p$ with

$$
\beta(p)=\frac{1}{n_{\mathrm{v}}} \sum_{\mathrm{p}_{\mathrm{v}} \in V(p)}\left\|I(p)-I\left(p_{\mathrm{v}}\right)\right\|
$$

$V(p)$ denotes the neighbors of $p$ and $n_{v}$ the cardinal of this set, $\|$.$\| is a L_{2}$-norm and $k$ is a real number which sets the fineness of the partition. $\beta(p)$ being close to a gradient computation, pixels in homogeneous regions (the color variation among the considered neighborhood is small) will be considered first as candidate region seeds. Each pixel is a candidate region 


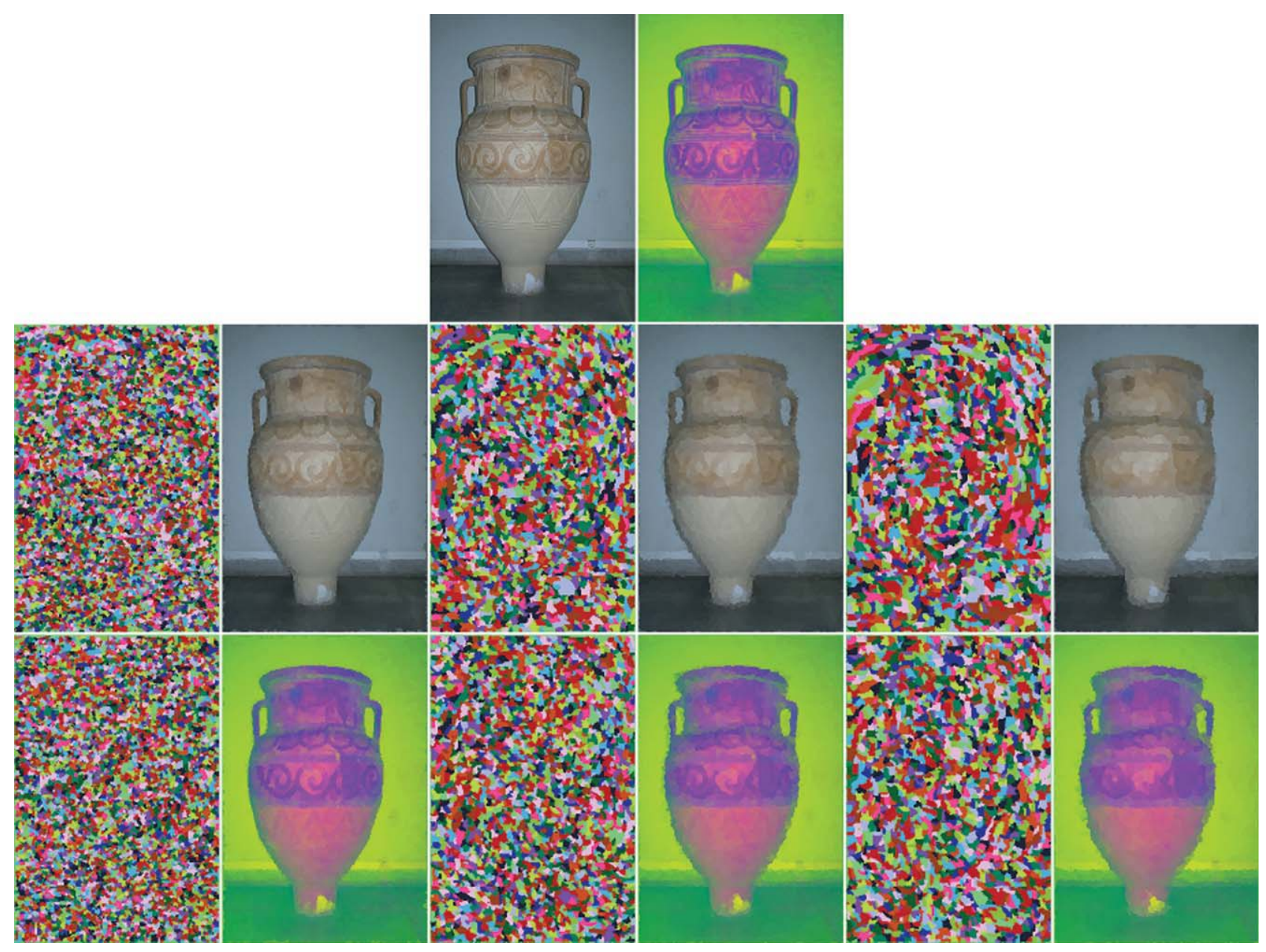

Figure 2. Hierarchies of partitions produced by the nonparametric hierarchical watershed at levels $1,10,20$. First line: initial image in RGB and $Y C_{b} C_{r}$, second line: RGB segmented images with theirs colorized (mosaic) images, third line: $Y C_{b} C_{r}$ segmented images with theirs colorized (mosaic) images. [Color figure can be viewed in the online issue, which is available at www.interscience.wiley.com.]

seed which grows by aggregating adjacent pixels according to the previous rule. This implies that a pixel $q$ is aggregated to a region $R$ if the distance between a pixel $p$ of $R$, neighbor of $q$, is $k$ times lower than the initial homogeneity of the seed pixel of $R . k$ is the accepted homogeneity jump and states if two pixels belong to the same region. Homogeneous zones therefore produce partitions, the fineness whose decreases while $k$ increases (the homogeneity constraint is slackened). Obviously, a hierarchy of partitions obtained for increasing values of $k$ is not nested as it lowers the number of initial seeds while slackening the homogeneity constraint. This is the same problem as producing nested partitions with the watershed: one has to consider the output of the $i$ th level as an input for the $(i+1)$ th level. Therefore, it is possible to produce hierarchical partitions using homogeneous connections by applying the same principle on the partition obtained at the previous level (an efficient implementation uses graphs). For that, we apply the principle of homogeneous connections on a RAG obtained by a fine partition of homogeneous connections. Each region of this RAG being described by its average, one can apply the same growth rule to the graph. We, therefore, obtain the principle of nested homogeneous connections described below.
Definition 8: Nested Homogeneous Connections (Meurie, 2005). Two nodes $p$ and $q$ of a RAG $G$ belong to same homogeneous zone of an image $I$ if $\left\|I\left(N_{p}\right)-I\left(N_{q}\right)\right\| \leq k \times \beta\left(\operatorname{Seed}\left(N_{p}\right)\right)$ with $\operatorname{Seed}\left(N_{p}\right)$ the seed node of the region of $N_{\mathrm{p}}$ with

$$
\beta\left(N_{\mathrm{p}}\right)=\frac{1}{n_{\mathrm{v}}} \sum_{N_{\mathrm{p}_{\mathrm{v}}} \in V\left(N_{\mathrm{p}}\right)}\left\|I\left(N_{\mathrm{p}}\right)-I\left(N_{\mathrm{p}_{\mathrm{v}}}\right)\right\|
$$

$I\left(N_{p}\right)$ denotes the average color of the pixels corresponding to the node $N_{p}, V\left(N_{p}\right)$ the neighbors of $N_{p}$ and $n_{v}$ the cardinal of this set. Each node $N_{p}$ of the graph is threaded in a hierarchical queue with the value of $\beta\left(N_{p}\right)$ as priority. The algorithm for the construction of nested homogeneous connections builds a hierarchy of partitions and needs two parameters $k$ and $k^{\prime} . k$ is a real number which sets the fineness of the base partition and $k^{\prime}$ the fineness of the next partitions of the hierarchy. The adjustment of $k$ and $k^{\prime}$ has a real importance in the performance of the algorithm. The algorithm of this method is described below. Homogeneous connections produce hierarchical partitions which are finer than the waterfall and coarser than the quasi flat zones. However, they are better suited in the case of automatic segmentation since they do not need a definition of 


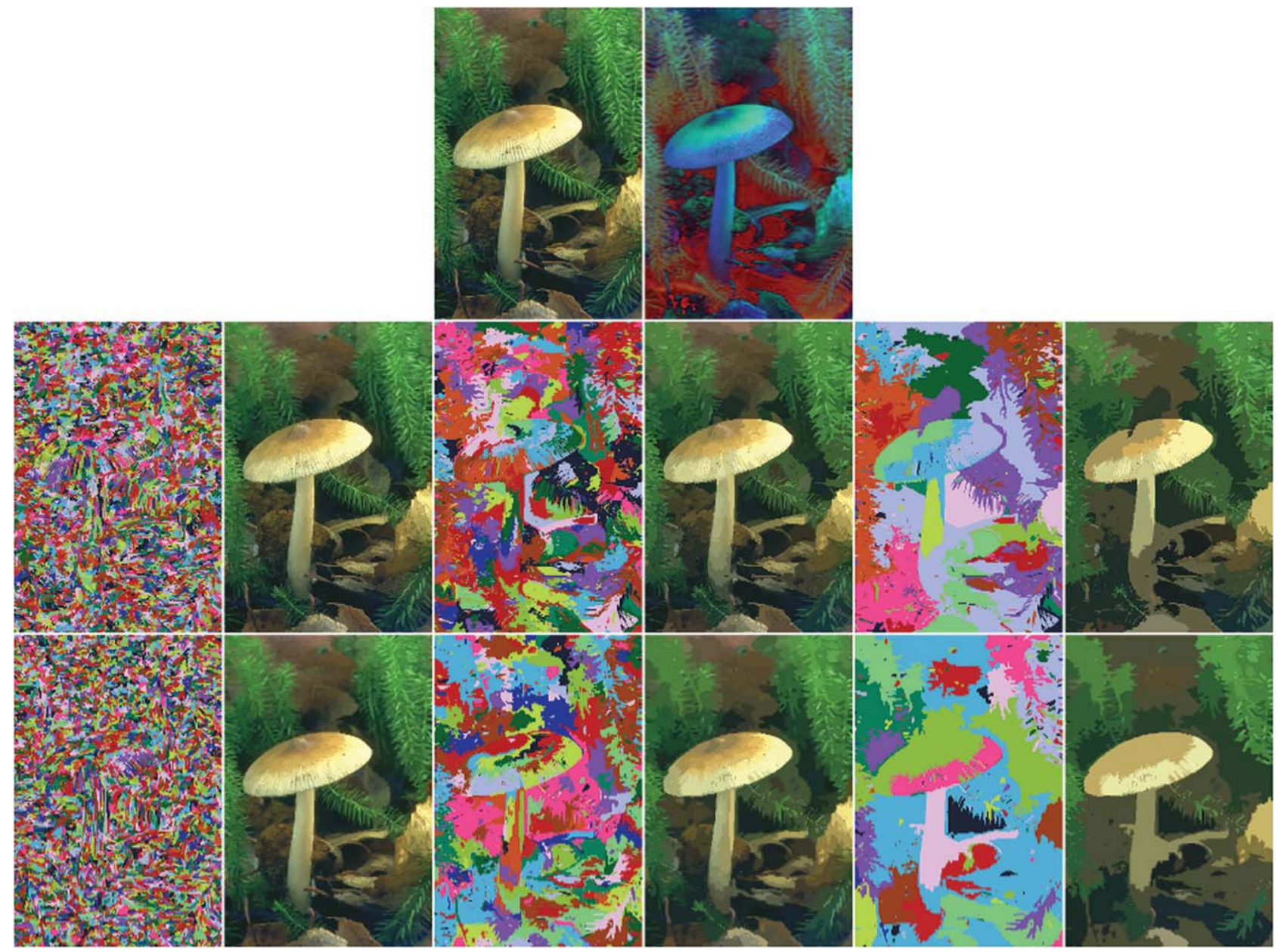

Figure 3. Hierarchies of partitions produced by the nested homogeneous connections at levels $1,5,15$. First line: initial image in RGB and IHSL, second line: RGB segmented images with their colorized images, third line: IHSL segmented images with their colorized images. [Color figure can be viewed in the online issue, which is available at www.interscience.wiley.com.]

seeds on a given gradient and they locally adapt their behavior to the image content since the threshold which determines if a pixel belongs to a region depends on the local homogeneity at this pixel.

Figure 3 provides an illustration of several hierarchical segmentations produced by the nested homogeneous connections algorithm for different levels of the hierarchy $(\beta \in\{1,5,15\})$ in two color spaces $(R G B, I H S L)$.

Let $\beta$ be an integer, $k$ and $k^{\prime}$ a real and $\beta_{\text {end }}$ a maximum number of iteration

1. $\beta \leftarrow 1$

2. $\mathrm{P}_{\beta} \leftarrow$ Homogeneous zones of $k$ fineness of the initial image

3. $G_{\beta}=\left(N_{\beta}, A_{\beta}\right)$ for an initial partition $\mathrm{P}_{\beta}$

4. while $\left(\beta \leq \beta_{\text {end }}\right)$ do

5. $\mid G_{\beta+1} \leftarrow$ Homogeneous zones of $k^{\prime}$ fineness of $G_{\beta}$

6. $\mid \beta \leftarrow \beta+1$

7. end while

IV. Experimental Results. In this section, we present an experimental study carried out on hundred images of the "Berkeley Segmentation Dataset and Benchmark (BSDB)." Results are shown in Figures 4-7. In Figures 4-6, the curves are obtained on the whole database (one hundred images of the BSDB). For this experimental study, the color space influence has been assessed on the above mentioned morphological hierarchical segmentation schemes: quasi flat zones, nonparametric hierarchical watershed, and the nested homogeneous connections. To evaluate the achieved results, objective criteria such as mean square error (MSE) has been considered. This objective quality measure can be expressed by the following equation:

$$
\text { MSE }=\frac{1}{N M} \sum_{i=1}^{N} \sum_{j=1}^{M}\left(o_{i, j}-x_{i, j}\right)^{2}
$$

where $o_{i, j}$ and $x_{i, j}$ respectively denote the original and reconstructed samples.

First, we consider the influence of the parameters $k$ and $k^{\prime}$ on the nested homogeneous connections. Results presented in Figure 4 show the evolution of the segmentation quality according to the increase of $k$ and $k^{\prime}$. Several values of $k$ are considered $(k \in\{0.5,1$, $1.5\}$ ), we recall here that the value of $k$ controls the fineness of the finest partition (the base partition of the further produced irregular pyramid). Therefore, for a given $k$, the segmentation quality decreases with the increase of the parameter $k$. For these different fineness levels of finest partition, all the corresponding nested homogeneous connections are extracted with $k^{\prime}$ ranging from 0.5 to 5 . The MSE objective quality criteria is computed for each level of 

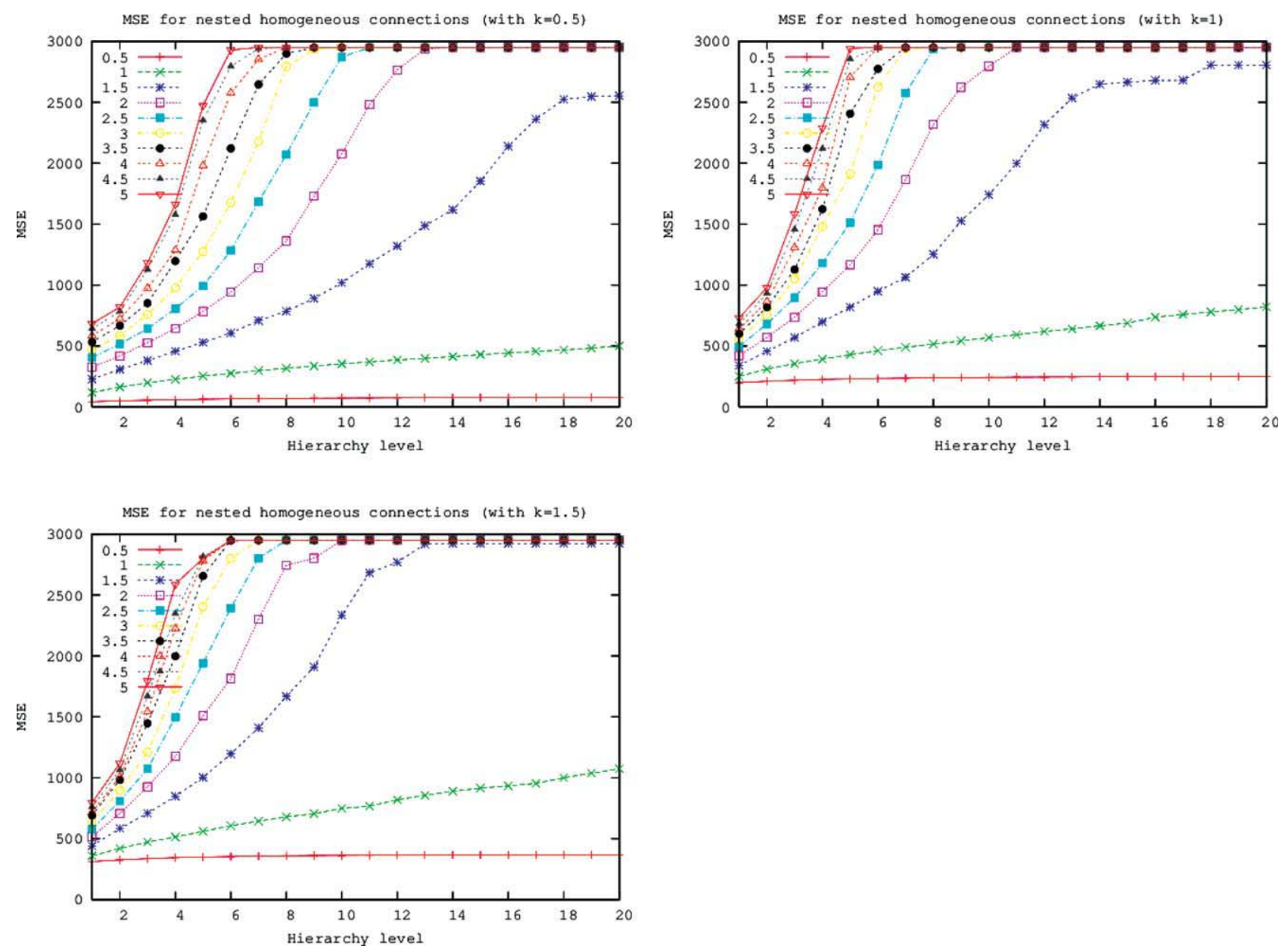

Figure 4. Evaluation with MSE of segmented images obtained by nested homogeneous connections with different parameters of $k$ and $k^{\prime}$. The used parameters are $k=0.5$ (top-left), $k=1$ (top-right), and $k=1.5$ (bottom-left). [Color figure can be viewed in the online issue, which is available at www.interscience.wiley.com.]

the hierarchy to assess the difference between the different values of the parameters. For this first experimentation, only the $R G B$ color space is considered. For the three tested values of $k$ and for $k^{\prime}$ upper to three, a saturation of the MSE in the first levels is observed which implies that the segmentation quality is poor and this finishes very fast in the hierarchy. It is therefore preferable to use a lower value of $k^{\prime}$ to increase the number of levels of the hierarchy which provides then a better multiscale representation of the image. A value of $k^{\prime}$ lower or equal to two seems to be a good compromise. The user can therefore choose the parameter $k^{\prime}$ within this range according to a compromise which can be visually or quantitatively assessed as a compromise between fidelity to the initial data and complexity of the involved model. Figure 5 presents several saliency maps of different hierarchical partitions obtained by nested homogeneous connections for $k$ ranging between 0.5 and 2 . The purpose of this illustration is to visually show the above remark on the level of the persistence of a pixel among the levels. A saliency map shows the importance of each pixel all along the levels of a hierarchy, the saliency of a pixel being defined as the highest level for which it occurs at the boundary between two regions (in the saliency map images, the gray level corresponds to the hierarchy level, that is to say the highest of the brightest). Indeed, the second image of Figure $5\left(k^{\prime}=0.5\right)$ has an important brightness since most of the regions always remain present along the hierarchy levels even for the highest. Such an hierarchical segmentation is therefore very close to the original content of the image but this involves a lot of levels to obtain interesting sole partitions of the image. On the opposite, there are much less regions in the last image $\left(k^{\prime}=4\right)$ which assesses the fact that for high values of $k^{\prime}$ very coarse hierarchical segmentations are obtained but they still extract the main visual features of the image. Usually, one would prefer to obtain a hierarchical segmentation which offers a good compromise between data fidelity (close to the initial image) and model complexity (few levels) as provided by the 3,4, and 5th images of Figure 5. To fix this compromise we chose $k=0.5$ and $k^{\prime}=1$ for the next experiments in this study. Once we have fixed the parameters of nested homogeneous connections, we consider the influence of color spaces on the production of morphological hierarchical partitions.

To end up the experimental studies, we consider the color space influence in terms of quantitative criteria. Figure 6 shows that, as expected, MSE increases as one progresses in a given hierarchy of partitions. This is inherent to the principle of hierarchical segmentation as progressing in the hierarchy means merging regions and conducting to a coarse segmentation. In Figure 6 (top-right), we can notice that the quasi flat zone scheme is very sensitive to the color space and the quality of the segmentation can highly vary from one color space to another. This is easy to explain. Quasi flat zones represents a threshold connection based on distances and both differ in 

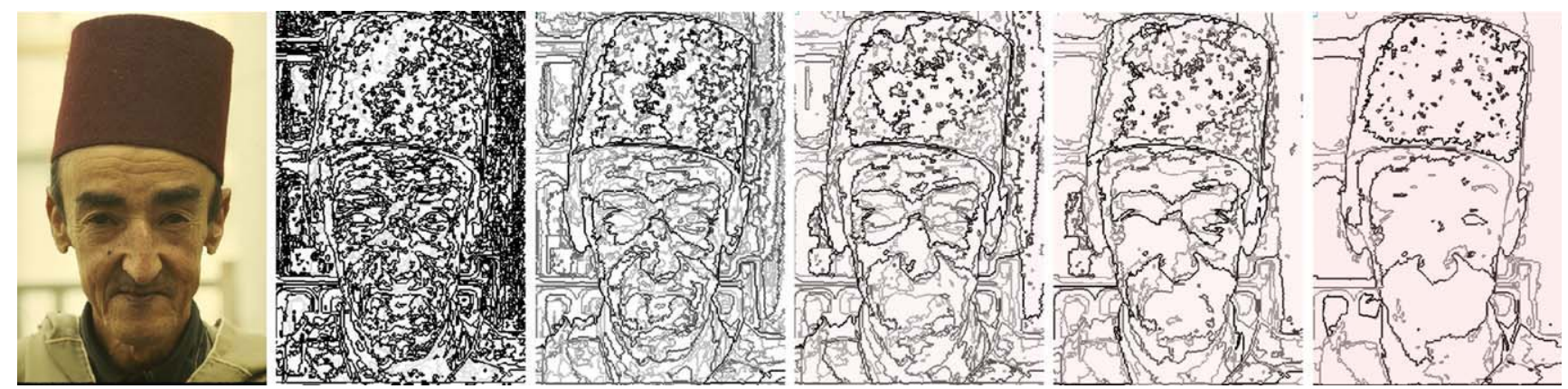

Figure 5. Saliency maps of different hierarchical partitions obtained by nested homogeneous connections with $k=1$ and different $k^{\prime}$ (from left to right: initial image, saliency maps with $k^{\prime}=0.5, k^{\prime}=1, k^{\prime}=1.5, k^{\prime}=2, k^{\prime}=4$ ). [Color figure can be viewed in the online issue, which is available at www.interscience.wiley.com.]
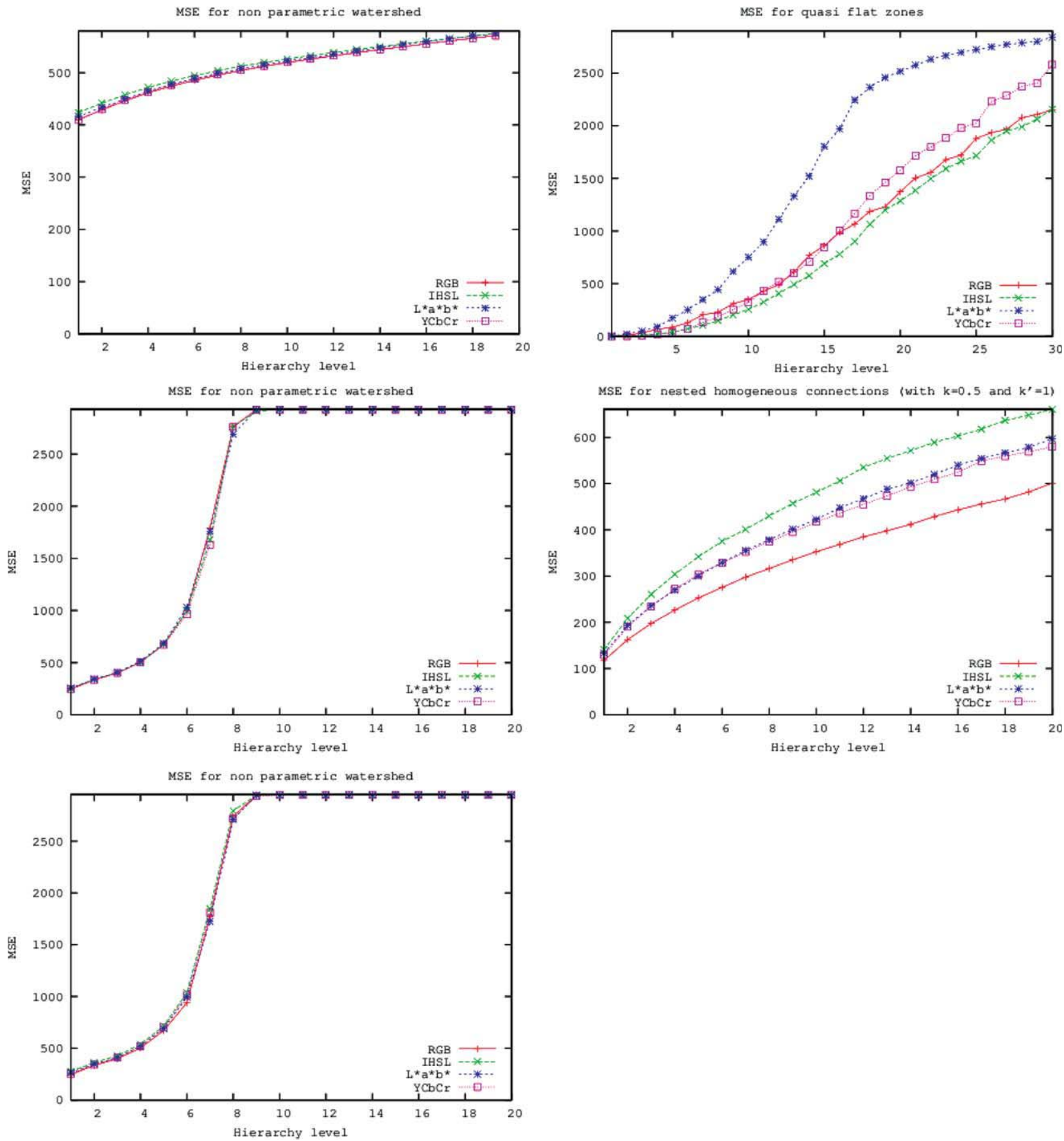

Figure 6. Evaluation with MSE of segmented images obtained by different methods of hierarchical segmentation in four color spaces (segmentation obtained by nonparametric hierarchical watershed with DiZenzo gradient (top-left), with vectorial morphological gradient (middle-left), with marginal morphological gradient (bottom-left), segmentation obtained by quasi flat zones [top-right), and nested homogeneous connections (middle-right)]. [Color figure can be viewed in the online issue, which is available at www.interscience.wiley.com.] 

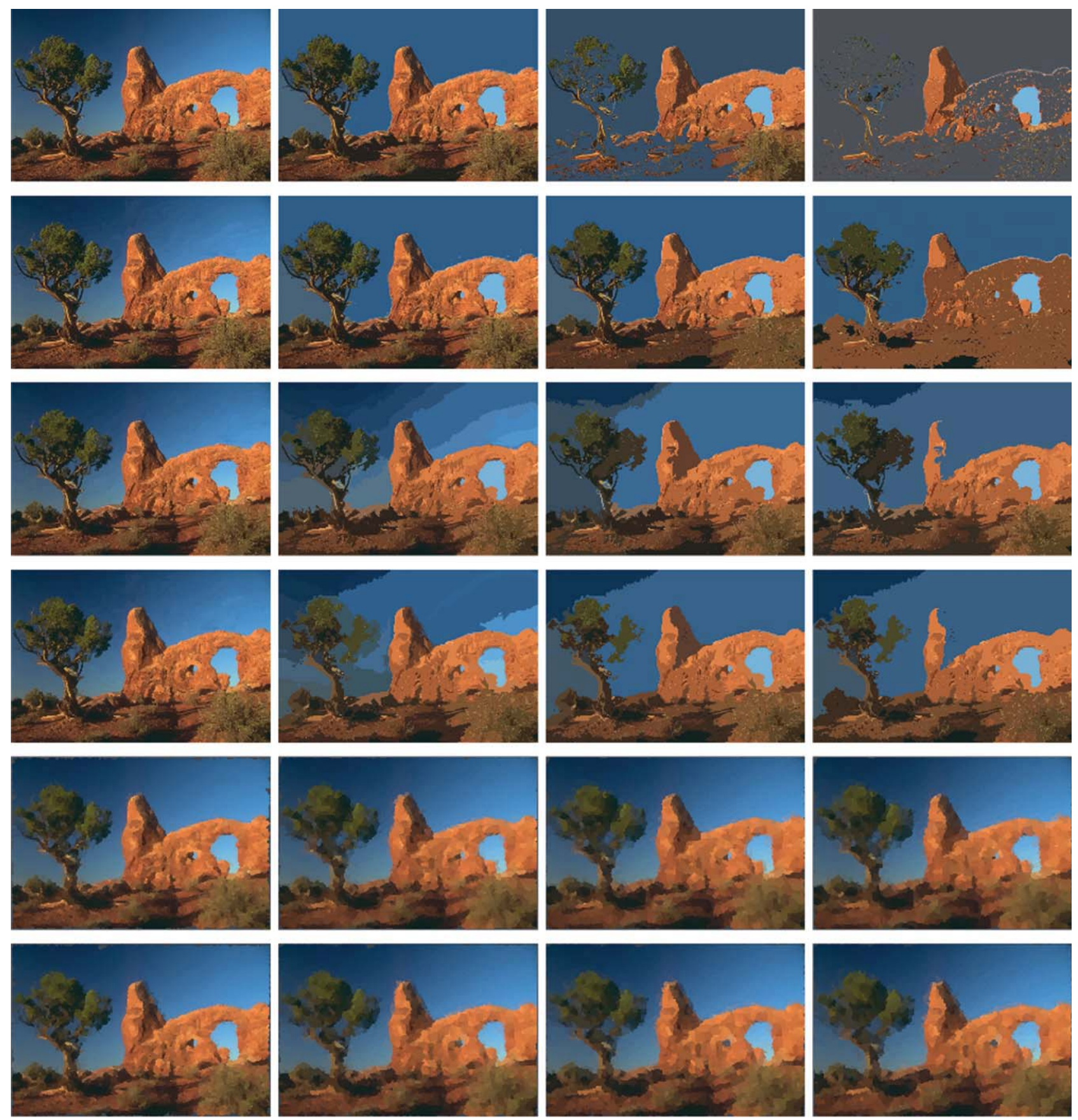

Figure 7. Initial and colorized images of partition hierarchies at levels 1 (column 1), 10 (column 2), 15 (column 3), 20 (column 4). The depicted schemes are quasi flat zones (lines 1 and 2), nested homogeneous connections (lines 3 and 4) and nonparametric hierarchical watershed with the DiZenzo (lines 5 and 6). Considered color spaces are RGB (lines 1, 3, 5) and IHSL color spaces (lines 2, 4, 6). [Color figure can be viewed in the online issue, which is available at www.interscience.wiley.com.]

different color spaces. Quasi flat zones are therefore absolutely not color space independent. It is also suitable to use the quasi flat zone in a color space given by the following preference ranking: $I H S L>R G B>Y C_{\mathrm{b}} C_{\mathrm{r}}>L^{*} a^{*} b^{*}$. On the opposite, as seen in Figure 6 (left), the color space has very little influence on the segmentation produced by the nonparametric hierarchical watershed. This result is not in compliance with previous results in the literature (Angulo, 2005) but we can explain it. In fact, the waterfall algorithm depends on the computing of a single input: the color gradient. For instance, in the reference works of Angulo, very few images are considered and even if a lot of different color morphological gradients are experimented (Angulo and Serra, 2003), their influence is only visually. And as one could expect it, they produce different results. To have a real quantitative evaluation on a consequent set of images, different gradients have been tested i.e., the general formulation of Di Zenzo (Fig. 6: top-left), a vectorial (Fig. 6: middle-left) and a marginal (Fig. 6: bottom-left) morphological gradients [see in (Angulo and Serra, 2003) for their formulations]. The conclusion is that the waterfall algorithm is quasi insensitive to the color space for a given color gradient. Moreover, there is no significant 

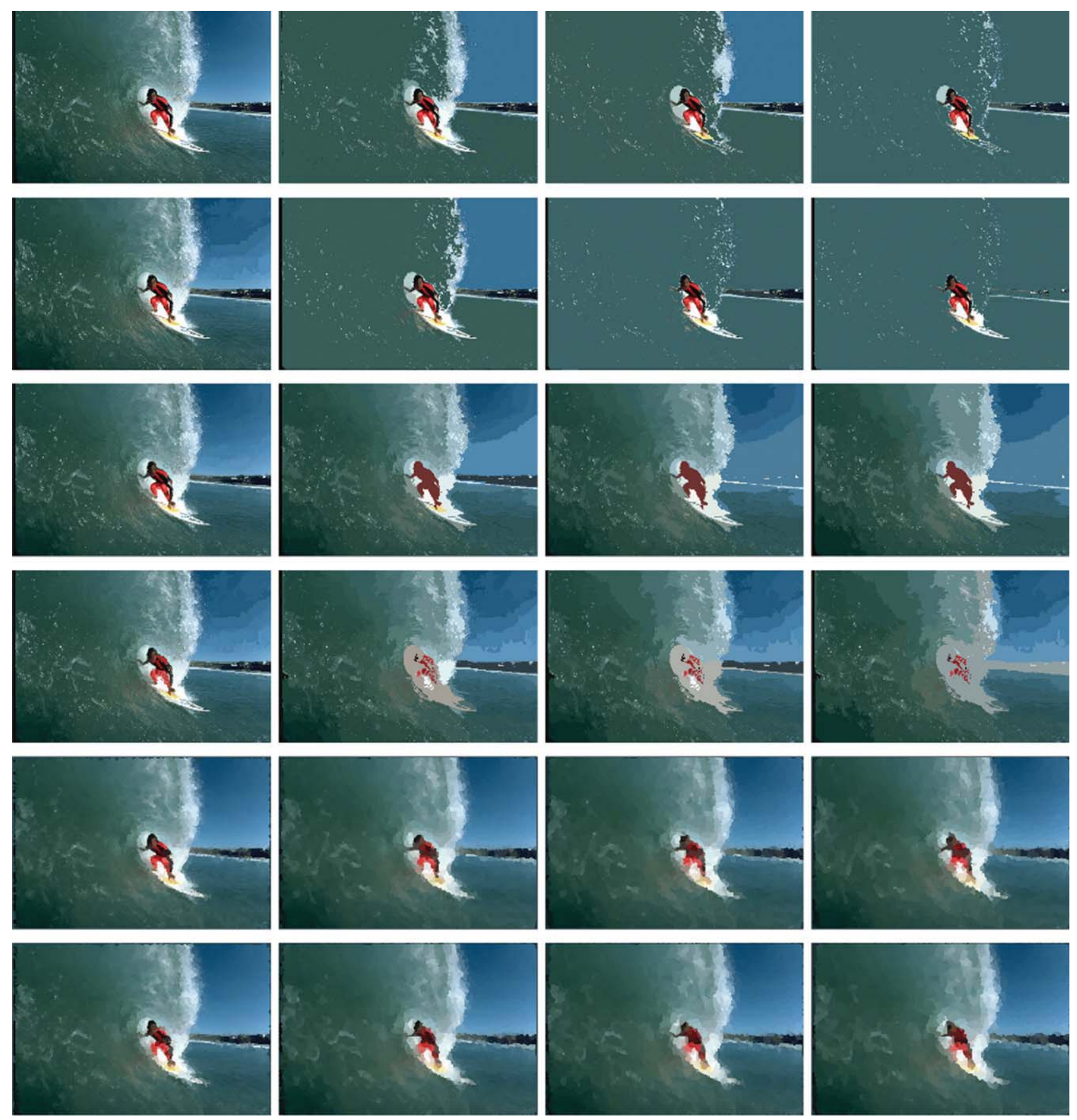

Figure 8. Initial and colorized images of partition hierarchies at levels 1 (column 1), 10 (column 2), 15 (column 3), 20 (column 4). The depicted schemes are quasi flat zones (lines 1 and 2), nested homogeneous connections (lines 3 and 4) and nonparametric hierarchical watershed with the DiZenzo (lines 5 and 6). Considered color spaces are RGB (lines 1, 3, 5) and IHSL color spaces (lines 2, 4, 6). [Color figure can be viewed in the online issue, which is available at www.interscience.wiley.com.]

difference between vectorial and marginal morphological gradients. However, the produced hierarchies with the Di Zenzo gradient contain a very huge number of levels and the produced models are not compact at all in comparison with the results produced by morphological gradients. Nested homogeneous connections illustrated in Figure 6 (middle-right) are a good intermediate connective criterion between pathwise (watershed and waterfall) and threshold connections (quasi flat zones) as regards the quality of the segmentation obtained (Meurie, 2005). As for the quasi flat zones, since a measure of distance is used, the color space has an influence. The later being however less pronounced than with the quasi flat zones, as a growing rule based on a measure of local homogeneity is used and not only a threshold. To conclude on this method, it is suitable to use the nested homogeneous connections in a color space given by the following preference ranking: $R G B>Y C_{\mathrm{b}} C_{\mathrm{r}}>L^{*} a^{*} b^{*}>I H S L$.

Figures 7 and 8 present several colorized (i.e., mosaic) images produced by the three hierarchical methods at different levels: 1 (column 1), 10 (column 2), 15 (column 3) and 20 (column 4). The depicted schemes are quasi flat zones (lines 1 and 2), nested homogeneous connections (lines 3 and 4) and nonparametric hierarchical 
watershed with the Di Zenzo gradient (lines 5 and 6). The best color spaces (i.e., $R G B$ for nested homogeneous connections and IHSL for quasi flat zones) are used to demonstrate the differences of the segmentation results. The images of Figures 7 and 8 stress out our previous remarks. For a given low hierarchy level (i.e., 1: column 1), the differences between the mosaic images produced by the hierarchical methods in $R G B$ (lines $1,3,5$ ) and IHSL (lines 2, 4, 6) color spaces is not really important. But that difference increases as one progresses in the hierarchy, expects, for the nonparametric hierarchical watershed which is insensitive to the color space. The extraction of these visual components being also different from one color space to the other. That is why, it is important to be careful to the choice of the color space for the quasi flat zones and the nested homogeneous connections. As previously explained, if we consider the quasi flat zones (lines 1 and 2), the best results are obtained in IHSL color space (line 2). For example, in $R G B$ color space (line 1), the tree and the soil are merged with the sky (Fig. 7: columns 3 and 4) and the surfboard are not well extracted (Fig. 8: columns 3 and 4). In contrast, to the nested homogeneous connections (lines 3 and 4), the best results are obtained in $R G B$ color space (line 3 ). The tree (Fig. 7: columns 3 and 4) and the surfer (Fig. 8: columns 3 and 4) are better extracted than in IHSL color space (line 4). These key examples permit to illustrate the prefered rankings (IHSL $>R G B>Y C_{\mathrm{b}} C_{\mathrm{r}}>L^{*} a^{*} b^{*}$ for quasi flat zones and $R G B>Y C_{\mathrm{b}} C_{\mathrm{r}}>L^{*} a^{*} b^{*}>I H S L$ for nested homogeneous connections) given earlier.

\section{CONCLUSION}

In this article, the color space influence on the production of partitions hierarchies of color images has been studied and assessed. The considered hierarchical partitions are morphological ones which rely on the use of a connective criterion. A threshold connection for color images is the quasi flat zones. A pathwise connection is the nonparametric hierarchical watershed (waterfall algorithm). A connective criterion is nested homogeneous connections which produces intermediate segmentations according to the two other methods (and so which are finer than the waterfall and coarser than the quasi flat zones). In terms of sensitivity to the different color spaces, the results show that the different methods do not have the same sensitivity. Quasi flat zones strongly react, nested homogeneous connections a little less and the nonparametric hierarchical watershed even less. To conclude, we can define a preference ranking of color spaces for two sensitive methods: $R G B>Y C_{\mathrm{b}} C_{\mathrm{r}}>L^{*} a^{*} b^{*}>I H S L$ for the nested homogeneous connections and $I H S L>R G B>Y C_{\mathrm{b}} C_{\mathrm{r}}>L^{*} a^{*} b^{*}$ for the quasi flat zones.

\section{REFERENCES}

J. Angulo, Unified morphological color processing framework in a lum/sat/ hue representation, In Proceedings of the International Symposium on Mathematical Morphology in Book: Mathematical Morphology 40 years on.'2005, C. Ronse, L. Najman and E. Decencière (Editors), Vol. 30. Springer-Verlag, Netherlands, 2005, pp. 387-396.

J. Angulo and B. Marcotegui, Sur l'influence des conditions d'éclairage dans la segmentation morphologique couleur de lpe, In Proc of Compression et Représentation des Signaux Audiovisuels (2005), 313-318.

J. Angulo and J. Serra, Color segmentation by ordered mergings, Proc ICIP 2003, Vol. 2, 2003, pp. 125-128.

V. Barnett, The ordering of multivariate data, J R Soc Stat 139 (1976), 318-355.

S. Beucher, Watershed, hierarchical segmentation and waterfall algorithm, Proceedings of International Symposium of Math Morphol Appl Image Signal Process (1994), 69-76.
Berkeley, The berkeley segmentation dataset and benchmark: BSDB, Available at http://www.cs.berkeley.edu/projects/vision/ grouping/segbench.

J. Chanussot and P. Lambert, Total ordering based on space filling curves for multivalued morphology, Proc fourth international symposium on mathematical morphology and its applications to image and signal processing, 1998, pp. 51-58.

M. Comer and E. Delp,, Morphological operations for colour image processing, J Elect Imaging, 8 (1999), 279-289.

J. Crespo, R. Schafer, J. Serra, C. Gratin, and F. Meyer, The flat zone approach: A general low-level region merging segmentation method, Signal Process 62 (1997), 37-60.

S. DiZenzo, A note on the gradient of a multi-image, Comput Vision Graph Image Process 33 (1986), 116-126.

A. Hanbury, A 3d-polar coordinate colour representation well adapted to image analysis, Proc SCIA, 2003, pp. 804-811.

S. L. Horowitz and T. Pavlidis, Picture segmentation by a tree traversal algorithm, J ACM, 23 (1976), 368-388.

A. Hanbury and J. Serra, Mathematical morphology in the HLS colour space, Proc BMVC'2001, Vol. 2, 2001a, pp. 451-460.

A. Hanbury and J. Serra, Morphological operators on the unit circle, IEEE Trans Image Process 10 (2001b), 1842-1850.

J.-M. Jolion and A. Montanvert, The adaptative pyramid, a framework for 2d image analysis, Comput Vision Graph Image Process 55 (1992), 339348.

W. Kropatsch, Building irregular pyramids by dual graph contraction, IEE Proc Vision Image and Signal Processing, Vol. 142, 1995, pp. 366374.

O. Lezoray, C. Meurie, P. Belhomme, and A. Elmoataz, A graph approach to color mathematical morphology, ISSPIT (IEEE Int Symp Signal Process Inform Technol), 2005a, pp. 856-861.

O. Lezoray, C. Meurie, P. Belhomme, and A. Elmoataz, Hierarchie de partitions pour la simplification et la segmentation d'images couleur, Compression et REprésentation des Signaux Audiovisuels, 2005b, pp. 231-236.

O. Lezoray, C. Meurie, P. Belhomme, and A. Elmoataz, Multi-scale image segmentation in a hierarchy of partitions, EUSIPCO CD Proc, 2006.

R. Lukac and K.N. Plataniotis, Color Image Processing: Methods and Applications, CRC Press/Taylor and Francis, FL, 2006.

C. Meurie, Color image segmentation by pixel classification and partition hierarchies, Ph.D. dissertation, University of Caen Basse-Normandie, 2005, France.

F. Meyer, From connected operators to levelings, Proc of International Symposium of Math Morphol Appl Image Signal Process (1998), 191-199.

F. Meyer, Hierarchies of partitions and morphological segmentation, Proc IEEE Workshop on Scale-Space and Morphology in Computer Vision and ICCV 2001, 2001a.

F. Meyer, An overview of morphological segmentation, Int J Pattern Recognit Artif Intelligence 15 (2001b), 1089-1118.

F. Ortiz, F. Torres, J. Angulo, and S. Puente, Comparative study of vectorial morphological operations in different color spaces, Proc Intelligent Robots and Computer Vision XX: Algorithms, Techniques, and Active Vision, Vol. 4572, (2001) pp. 259-268.

R.A. Peters, Mathematical morphology for angle valued images, SPIE International Conference on Electronic Imaging, Vol. 3026, 1997, pp. 84-94.

I. Pitas and P. Tsalides, Multivariate ordering in color image filtering, IEEE Transactions on Circuits and Systems for Video Technology, Vol. 1, 1991, pp. 247-259.

K. Plataniotis and A. Venetsanopoulos, Colour Image Processing Handbook, Chapman and Hall, 1998, pp. 188-209, London, New York. 
C. Ronse, Why mathematical mophology needs complete lattices, Signal Processing 21 (1990), 129-154.

P. Salembier and J. Serra, Morphological multiscale image segmentation, SPIE Visual Communications and Image Processing, 1992, pp. 620-631.

J. Serra, Connections for sets and functions, Fundam Inform 41 (2000), 147186.

J. Serra, Morphological segmentations of colour images, Proc International Symposium on Mathematical Morphology in Book: Mathematical Morphology 40 years on'2005, C. Ronse, L. Najman, and E. Decenciere (Editors), Vol. 30. Springer-Verlag, Netherlands, 2005, pp. 151-176.

J. Serra, A lattice approach to image segmentation, J Math Imaging Vision 24 (2006), 80-130.

G. Sharma and H.J. Trussell, Digital color imaging, IEEE Trans Image Processing 6 (1997), 901-932.

H. Talbot, C. Evans, and R. Jones, Complete ordering and multivariate mathematical, Proc Fourth international symposium on Mathematical morphology and its applications to image and signal processing, 1998, pp. 27-34.

P. Tsalides, M. I. Vardavoulia, and I. Andreadis, Vector ordering and morphological operations for colour image processing, Pattern Anal Appl 5 (2002), 271-287.

N. Vandenbroucke, Segmentation d'images couleur par classification de pixels dans des espaces d'attributs colorimétriques adaptés. Application à l'analyse d'image de football, Ph.D. dissertation, University of Lille 1, 2000, France.

N. Vandenbroucke, L. Macaire, and J. G. Postaire, Color systems coding for color image processing, Proc Int Conf on Color in Graphics and Image Processing, Vol. 1, 2000, pp. 180-185.

G. Wyszecki and W.S. Stiles, Color science: concepts and methods, quantitative data and formulae, 2nd edition, Wiley, New York, 1982.

S. Zucker, Region growing: Childhood and adolescence, Comput Graph Image Process 5 (1976), 382-399. 